\title{
Prevalence, Pathogenesis, Diagnosis, and Management of Microscopic Colitis
}

\author{
Nicole Gentile and Eugene F. Yen \\ NorthShore University HealthSystem, University of Chicago, Evanston, IL, USA
}

Microscopic colitis (MC), which is comprised of lymphocytic colitis and collagenous colitis, is a clinicopathological diagnosis that is commonly encountered in clinical practice during the evaluation and management of chronic diarrhea. With an incidence approaching the incidence of inflammatory bowel disease, physician awareness is necessary, as diagnostic delays result in a poor quality of life and increased health care costs. The physician faces multiple challenges in the diagnosis and management of $\mathrm{MC}$, as these patients frequently relapse after successful treatment. This review article outlines the risk factors associated with MC, the clinical presentation, diagnosis and histologic findings, as well as a proposed treatment algorithm. Prospective studies are required to better understand the natural history and to develop validated histologic endpoints that may be used as end points in future clinical trials and serve to guide patient management. (Gut Liver 2018;12:227-235)

Key Words: Colitis, microscopic; Colitis, lymphocytic; Colitis, collagenous

\section{INTRODUCTION}

Microscopic colitis (MC), comprised of lymphocytic colitis (LC) and collagenous colitis (CC), is a clinicopathologic diagnosis commonly encountered in practice for the evaluation and management of chronic diarrhea. With the incidence approaching that of inflammatory bowel disease (IBD), physician awareness is necessary as diagnostic delays result in poor quality of life and increased health care costs. However, clinicians face multiple challenges associated with the diagnosis and management of MC due to the lack of robust evidence and societal guidelines for induction and long term maintenance therapy. In part, this may be due to our lack of understanding of histologic and clini- cal predictors of disease severity to help better inform clinicians of the natural history of MC. Although our awareness of this condition has increased, prospective studies are needed to understand the natural history, validate histologic endpoints, and better define treatment algorithms which will impact both clinical care and serve as end points for clinical trials.

\section{EPIDEMIOLOGY}

Since the first description in 1985, the incidence of MC has increased with rates approaching that of other forms of IBD, based on epidemiologic studies conducted in Spain, Europe, North America. ${ }^{1-6}$ In a population based study of Olmsted County, United States, from 1985 to 2001, the prevalence of MC was 103.0 per 100,000 person-years, demonstrating an overall increase during that time period. ${ }^{4}$ This population based cohort was updated to examine the incidence trend from 2002 to 2010 which demonstrated disease stability with an overall incidence of 21.0 per 100,000 person-years. ${ }^{3}$ This is comparable to a Danish cohort with an overall incidence in 2011 of 24.7 per 100,000 inhabitants. ${ }^{5}$ A recent meta-analysis has demonstrated the overall incidence of CC 4.14 (95\% confidence interval [CI], 2.89 to 5.40) per 100,000 person-years and 4.85 for LC ( $95 \%$ CI, 3.45 to 6.25). ${ }^{7}$ Again, similar to the results of the Olmstead cohort, the authors show that the temporal trend of increasing incidence prior to 2000, which stabilized thereafter. ${ }^{7}$ Multiple trials have demonstrated the association of MC with women and older age. $^{3,6,7}$

\section{RISK FACTORS}

\section{Medications}

The association between the intake of medications and the development of MC is a controversial entity. Some believe that

\footnotetext{
Correspondence to: Eugene F. Yen

NorthShore University HealthSystem, University of Chicago, 2650 Ridge Avenue, Suite G221, Evanston, IL 60201, USA

Tel: +1-847-570-3890, Fax: +1-847-733-5041, E-mail: eyen@northshore.org

Received on February 9, 2017. Revised on March 24, 2017. Accepted on March 24, 2017. Published online July 4 , 2017 pISSN 1976-2283 eISSN 2005-1212 https://doi.org/10.5009/gnl17061

(c) This is an Open Access article distributed under the terms of the Creative Commons Attribution Non-Commercial License (http://creativecommons.org/licenses/by-nc/4.0) which permits unrestricted non-commercial use, distribution, and reproduction in any medium, provided the original work is properly cited.
} 
medications are causal whereas others argue that MC may result in an insult to the colon causing symptoms to present sooner. We hypothesize the latter is more likely and has biologic plausibility. It is known that both proton pump inhibitors (PPIs) and nonsteroidal anti-inflammatory drugs (NSAID) medication may affect colonic permeability allowing for passage of antigens into the lamina propria which may cause as an immune reaction leading to a lymphocytosis. ${ }^{8,9}$ However, evidence-based literature has not demonstrated a distinct causation, rather associations have been implied.

A proposed scoring system was developed by Beaugerie et al. ${ }^{10}$ who suggested that certain medications, based on review of case reports in the literature, were associated with a high likelihood of causing MC. A case control study comparing those with CC $(n=39)$, LC $(n=39)$, and watery diarrhea without a diagnosis of MC ( $n=52)$ found a statistical association between LC and selective serotonin reuptake inhibitors (SSRIs), $\beta$-blockers, HMGCoA reductase inhibitors (statins), and bisphosphonates, with an increased association of NSAIDS and SSRIs in those with CC. ${ }^{11}$ Furthermore, in a nationwide Danish case control study with 5,751 cases, an association between PPI and LC (adjusted odds ratio [OR], 3.37; 95\% CI, 3.08 to 3.69) and CC (adjusted OR, 7.04; 95\% CI, 6.55 to 7.56), SSRI and LC (adjusted OR, 2.89; 95\% CI, 2.61 to 3.20), and SSRI and CC (adjusted OR, 1.59; 95\% CI, 1.45 to 1.79 ) was demonstrated. ${ }^{12}$ Ultimately, all four classes of medication (PPI, SSRI, NSAID, and statin) were associated with a diagnosis of MC. ${ }^{12}$

Drug induced colitis is a separate clinical entity, which poses a challenge as patients with MC may also have concurrently taken NSAIDs at time of MC diagnosis. In a recent study, the degree of mucosal eosinophilia was investigated as it related to medication. ${ }^{13}$ Histologically, the difficulty is that NSAID colitis may present as a nonspecific pattern and appear histologically similar to IBD or CC. ${ }^{13,14}$ These authors ultimately concluded that left sided colonic increase in mucosal eosinophilia may be suggestive of a drug induced colitis as healthy adults generally have less than 3 eos/hpf. ${ }^{13}$ According to their findings, drug induced colitis lacked the histologic hallmarks of CC (thickened subepithelial collagen band, dense lamina propria infiltration, and increased intraepithelial lymphocytosis) as well as the patients with CC generally have a history of chronic diarrhea. ${ }^{13}$ Interestingly, whereas eosinophils are generally located throughout the colon with CC, in drug induced colitis eosinophils are left side colon predominant. ${ }^{13}$ Increased degrees of eosinophil infiltration in patients with CC may be due to eosinophil degranulation resulting in a subepithelial collagen band and epithelial cell injury. ${ }^{15}$

With limited retrospective data available, it is also possible that medication induced colitis is a separate entity from MC as clinical experience has shown that in a subset of patients who discontinue their medication (i.e., NSAIDs), the diarrhea resolves without further intervention. Prior studies are limited because they are retrospective and do not address if there are any histologic differences in patients on medications at time of MC diagnosis versus those with MC not on medications. Furthermore, conclusions of these retrospective studies are not always representative of clinical experience with data on disease severity and correlation lacking.

\section{Risk factors: smoking}

Cigarette smoking has an established relationship with IBD. ${ }^{16}$ Its association with $\mathrm{MC}$ has also recently been proposed. In a study of 340 patients with MC, smoking was a risk factor in disease development in both men and women with an adjusted OR of 4.37 for current smokers (95\% CI, 2.43 to 7.86). ${ }^{17}$ Furthermore, in an observational study 100\% of those with CC had a smoking history prior to disease development. ${ }^{18}$ In addition, authors found that those who were current smokers developed CC at an earlier age $(\mathrm{p}<0.003) .{ }^{18}$ The proposed association may be related to decrease in colonic blood flow caused by smoking as well as decrease in mucosal permeability. ${ }^{16}$ Thus, we currently discuss and advise smoking cessation in our patients with MC.

\section{Clinical presentation}

Patients with MC often present with chronic nonbloody diarrhea and tend to be a female of older age. ${ }^{3}$ There is also an association with other autoimmune disease including rheumatoid arthritis, psoriasis, thyroid disease, and type I diabetes. ${ }^{19,20}$ Patients may also present with nonspecific symptoms include abdominal pain, weight loss depending on severity of diarrhea, fecal incontinence, urgency, and fatigue. ${ }^{1,19-22}$ The symptom presentation of both LC and CC are similar. ${ }^{23} \mathrm{MC}$ is generally a chronic condition with diarrheal severity ranging from a few bowel movements per day to $>10$ bowel movements per day, nocturnal stools, and disease relapses. ${ }^{23}$ As patients present with nonspecific symptoms, the presentation may initially be confusing with irritable bowel syndrome (IBS). A recent meta-analysis compared IBS to MC demonstrated a prevalence of 33.4\% of IBS symptoms in patients with MC; however, this was similar to patients with diarrhea and without a diagnosis of MC. ${ }^{24}$ In a cohort comparison between patients with MC and IBS, 40\% met Rome I criteria, 38\% met Rome II criteria, and 27\% who had been diagnosed as IBS were determined to have MC on colon biopsy. ${ }^{25}$ Prospective studies have compared patients with functional diarrhea to MC and found a higher risk for MC in females, age $>50$ years, those with autoimmune disease, and symptoms of weight loss or nocturnal stools. ${ }^{26}$ In these patients with alarm features and chronic diarrhea we agree with the author's recommendation for colonoscopy with biopsy.

An additional challenge in patient management is the lack of a standardized definition of disease severity and thus impact on treatment. As well, clinical endpoints of disease remission have not been clearly defined. A case control study of patients with MC with a median disease duration of 5.9 years (for CC) 
and 6.4 years (for LC) was the first study to show persistence of symptoms despite remission. ${ }^{21}$ Patients with CC had persistent abdominal pain, fatigue, arthralgia, and myalgia whereas those with LC also had persistent fatigue $(\mathrm{p}<0.05){ }^{21}$ Generally, resolution of diarrhea has been used as an endpoint, however the authors propose the above symptoms also are included when determining which patients are in remission. ${ }^{21}$

\section{Disease severity}

Given the symptom overlap with IBS and the lack of histologic criteria to stratify severity, it is difficult to assess disease activity to guide therapy. Thus, quality of life has been used as a surrogate measure to determine severity. In a cross sectional survey of 116 patients with CC, the association between quality of life and stool frequency was studied. ${ }^{27}$ Symptoms including urgency, number of stools per day including watery stools per day, and abdominal pain were assessed and compared to four indices of quality of life including symptom burden, social function, disease related worry and general well-being. ${ }^{27}$ Clinical remission was defined as $<3$ stools per day and $<1$ watery stool per day, based on the findings that those with $\geq 3$ stools per day or a mean of $\geq 1$ watery stool per day had the greatest impact overall on poor quality of life. ${ }^{27}$ Moreover, those who reported $\geq 3$ stools per day also had associated urgency (74\%) and abdominal pain (89\%). ${ }^{27}$ Until recently, no objective parameters have been defined. The MC Disease Activity Index found four independent clinical features including number of unformed stools daily, number of nocturnal stools, abdominal pain, and number of episodes of fecal incontinence that correlated with a physician's global assessment. ${ }^{28}$ As limited information is available, more studies are needed to help predict the natural history of disease to guide treatment decisions, as well as serve as a more rigorous endpoint for clinical trials.

\section{PATHOPHYSIOLOGY}

\section{Mechanism of diarrhea}

The mechanism of diarrhea (Table 1) in the cause of MC remains largely unknown, partly due to lack of animal models. ${ }^{29}$ As far back as 1985, the steady state perfusion of the colon of patients with MC was evaluated and concluded that "reduced active sodium and chloride absorption, inhibition of chloride/ bicarbonate exchange, and decreased passive permeability of the mucosa" may be the result of an inflammatory process. ${ }^{30}$ Sodium chloride absorption in biopsies of patients with CC has been further studied at the molecular level. ${ }^{31}$ Ultimately, researchers found that there was a decrease in both $\mathrm{Na}^{+}$and $\mathrm{Cl}^{-}$absorption, epithelial resistance, occludin and claudin-4 expression, regulation of tight junction molecules, resulting in a dysfunction of the collagen barrier and ultimately, leading to a secretory diarrhea. ${ }^{31}$ Theories of a secretory diarrhea is also supported by production of Prostaglandin E2 by pericryptal fi- broblasts. ${ }^{32}$ Although, multiple studies support a secretory component there are studies that demonstrate an osmotic element of the diarrhea. Electrolyte disturbances have been documented in 76 patients with MC with $86.7 \%$ having secretory diarrhea and the remaining an osmotic diarrhea. ${ }^{33}$ The osmotic theory is supported where investigators noted that after a period of fasting, fecal weight decreased in 14 patients with $\mathrm{CC}^{34}$ Fecal weight has also been associated with lamina propria inflammation and cellularity $^{35}$ and the European Consensus Group postulates that the severity of the diarrhea may be related to this microscopic inflammatory change. ${ }^{19}$

Serotonin has an established role in ulcerative colitis and impacts both motility and luminal secretion. ${ }^{29}$ There is evidence that a polymorphism of a serotonin reuptake transporter may play a role in the cause of MC. ${ }^{29}$

Another mechanism of diarrhea involves bile acid malabsorption. Bile acid sequestrants such as cholestyramine have been used with varying success in some patients with MC. Therefore it is plausible that bile salts impact colonic absorption and contribute to the diarrhea as well as some patients with MC may have ileal involvement. ${ }^{33}$ Selenium labeled homocholic acid taurine test $\left({ }^{75} \mathrm{SeHCAT}\right)$ has been used to study bile acid malabsorption. ${ }^{36}$ In 27 patients with CC, $44 \%$ had an abnormal ${ }^{75}$ SeHCAT test and ultimately 78\% (21/27) of the CC patients had improvement in their diarrhea with treatment of cholestyramine. ${ }^{36}$

Inflammatory mediators in the lamina propria resulting in surface injury have also been proposed as a cause of diarrhea. ${ }^{37}$ A luminal component is supported as observation of a small number of patients who have undergone a diverting ileostomy demonstrate histologic improvement in their MC, which returns upon reconnection and resumption of the fecal stream. $.^{20,38,39} \mathrm{Ex}-$ posure to a luminal antigen (medication, dietary, bile salts, bacteria) may cause an inflammatory cascade due to activation of CD8 $\mathrm{T}$ suppressor cells that may directly attack and damage the enterocyte. ${ }^{23,33}$ Further, exposure to a luminal antigen is believed to stimulate transcription factor nuclear- $\kappa \beta$ resulting in induc-

Table 1. Proposed Mechanism of Diarrhea

Mucosal permeability changes due to inflammation
Decreased sodium and chloride absorption
Inhibition of chloride and bicarbonate exchange ${ }^{31}$
Changes in epithelial resistance ${ }^{31}$
Production of Prostaglandin $\mathrm{E}^{32}$
Polymorphism of a serotonin reuptake transporter ${ }^{29}$
Impact on motility and secretion
Bile acid malabsorption
Inflammatory mediators in the lamina propria
Luminal antigens (medication, dietary, bile salts, bacteria) $^{33}$
Activation of CD8 $\mathrm{T}$ suppressor cells and transcription factor
nuclear- $\kappa \beta^{23,33,40}$


ible nitric oxide synthase gene expression which increases nitric oxide levels. ${ }^{40}$ This pathway was studied in vivo in patients with CC and concluded that "downstream inflammatory reactions leading to tissue damage original in lamina propria of immune cells, as increased transcription factor nuclear $\kappa \beta$ activity in CC was localized solely in epithelial cells." ${ }^{40}$ Further, studies have demonstrated symptomatic improvement in patients treated with antimicrobials as well as described disease onset following infection. ${ }^{23,41}$ In a retrospective study, MC patients received treatment with metronidazole and erythromycin with clinical responses of 55\% and 67\% supporting an antimicrobial cause of diarrhea. ${ }^{41}$ In this same study, 19 of 163 were treated with mepacrine hydrochloride which possess antimicrobial properties as well as inhibits arachadonic acid, with approximate 50\% responding to treatment. ${ }^{41}$

\section{Association with celiac disease}

There is a proposed association between MC and celiac disease (CD), with an increased risk of 50- to 70-fold compared to the general population. ${ }^{20,42-48}$ In a population based study from 2004 to 2008, 40 out of 120 patients who had both duodenal and colonic biopsies were diagnosed with both MC and CD; $90 \%$ were female with a mean age of 50.1 years of age. ${ }^{42}$ This association was also demonstrated in a prospective cohort of patients with established CD (1981 to 2006), of which MC was diagnosed in 4.3\% of these CD patients who tended to be older and have more severe villous atrophy on histology. ${ }^{43}$ When compared to the general population, those with CD where at 70fold increased risk of developing MC with 64\% diagnosed with MC subsequent to CD diagnosis, 11\% with MC first, and 25\% both concomitantly diagnosed. ${ }^{43}$

CD is associated with the human leukocyte antigen (HLA) class II genes, particularly HLA DQ2 and HLA DQ8 alleles. ${ }^{45,47}$ Greater than " $90 \%$ of those with CD possess the HLA DR3 or DR5/7 combination associated DQ2 molecule encoded by DQA1*0501 and DQB1*02 allele with most of the rest having HLA DR4-DQ8 (encoded by DQA1*03 and DQB1*0302)." ${ }^{* 4}$ Up to $30 \%$ of the Caucasian population may test positive for HLA DR3 DQ2, and less than 10\% will be diagnosed with $\mathrm{CD} .{ }^{45}$ As $\mathrm{CD}$ is felt to be an autoimmune condition, some hypothesize an autoimmune component to MC as studies have shown positivity for HLA DQ2 in both diseases. ${ }^{45}$

To better define the potential for a shared genetic predisposition, patients with $\mathrm{MC}, \mathrm{CD}$, and normal controls were studied for the presence of HLA DQ2 genes. ${ }^{47}$ HLA DQ2 typing was positive in $88 \%$ with CD and 64\% with MC compared to $31 \%$ of controls. ${ }^{47}$ The presence of HLA DQ2 types was also described in an additional study of 80 patients with MC (29 CC, 51 LC). ${ }^{45}$ Of these patients, 78 of 80 also had duodenal biopsies. ${ }^{45}$ The HLADR3-DQ2 was positive in 43.8\% with MC overall, $44.8 \%$ with CC, $43.1 \%$ with LC, and $86.7 \%$ of those with both MC and CD diagnoses compared to a control group of $18.1 \%{ }^{45}$ HLA-DR4-
DQ8 was positive in 13.8\% with MC overall, 13.8\% with CC, 13.7\% with LC, 6.7\% with concomitant CD and MC compared to a control group of $23.0 \% .^{45}$ of those who had duodenal biopsies for CD ( $\mathrm{n}=78)$, CD was diagnosed in 15 of 80; 11 were diagnosed prior to $\mathrm{MC}$, three diagnosed concomitant at time of MC diagnosis, and one diagnosed with $\mathrm{CD}$ after a diagnosis of MC. ${ }^{45}$ Ultimately authors conclude there is an association with the HLA-DR3-DQ2 haplotype with CC and LC in patients with MC with and without $\mathrm{CD} .{ }^{45}$ Similarly, in a prospective study of 59 patients with MC compared to healthy population controls the HLA DQ2 allele was demonstrated in $48 \%$ with LC, 32.3\% of those with CC, and $24.3 \%$ of controls with no overall difference with the HLA DQ8 allele. ${ }^{46}$ Thus, these studies support that there may be a genetic overlap in the HLA DQ genes in those with MC, CD, and MC with CD; however, the association with HLA DQ8 is weak.

The presence of celiac serology autoantibodies in patients with only MC has also been evaluated. In a prospective study of 59 patients with MC compared to healthy population controls $(\mathrm{n}=70)$ blood tests for HLA-DQ2, HLA-DQ8, serum immunoglobulin A antiendomysial antibody (AEA), serum IgA-human antitissue transglutaminase antibodies (tTGA) were done. ${ }^{46}$ Twentythree patients with HLA DQ2 or DQ8 positivity underwent duodenal biopsies (13 with CC and 10 with LC) which showed Marsh I classification in four of $23 .^{46}$ Of these four, 75\% were treated and responded to a gluten-free $\operatorname{diet}^{46}$ Of the 59 patients who underwent serologic testing, none were IgA deficient, and 98\% were negative for AEA and tTGA. ${ }^{46}$ One patient was identified with LC who was positive for DQ2, AEA, tTGA, and biopsy consistent with Marsh I. ${ }^{46}$ Studies have also evaluated the presence of antiendomysial and antigliadin antibodies in those with MC. ${ }^{47}$ In patients with $\mathrm{CD}(\mathrm{n}=25)$, antigliadin IgA was positive in $76 \%$ and mean AEA titer was $>1: 32$; this was compared to those with MC only ( $n=53)$ who had a positive antigliadin IgA in 11\% and a mean AEA titer of $>1: 7.5$ (normal value is $<1: 5$ ). ${ }^{47}$ Given this limited data, further larger prospective studies are needed to determine the significance of celiac serology autoantibodies in those with MC.

Lastly, genome wide assay analysis has also been studied in those with CD. A study of 1,550 patients with CD and 3,084 controls SNPs was studied. Of the 1,550, 4.5\% had MC identified on clinical documentation in the medical record or pathology. They found similar SNPs 3p21.31, 6q15, 6q25.3, and 1q24.3 in those with both CD and MC further suggesting an association. ${ }^{48}$ This association is clinically relevant, particularly in the patient with MC who presents with refractory diarrhea despite treatment, in which evaluation for concomitant CD is recommended.

\section{DIAGNOSIS}

The diagnosis of MC involves the combination of clinical presentation with histologic changes. Generally, a patient will 
Table 2. Histologic Definition of Lymphocytic Colitis and Collagenous Colitis

\begin{tabular}{ll}
\hline & \multicolumn{1}{c}{ Histology } \\
\hline Lymphocytic colitis $^{1,50-53}$ & Presence of intraepithelial lymphocytes (>20/100 colonic surface epithelial cells) \\
& Normal colonic architecture \\
Collagenous colitis $^{54}$ & Presence of an abnormally thickened subepithelial collagen band $>10 \mu m$ \\
& Increased intraepithelial lymphocytes \\
& Normal colonic architecture \\
\hline
\end{tabular}

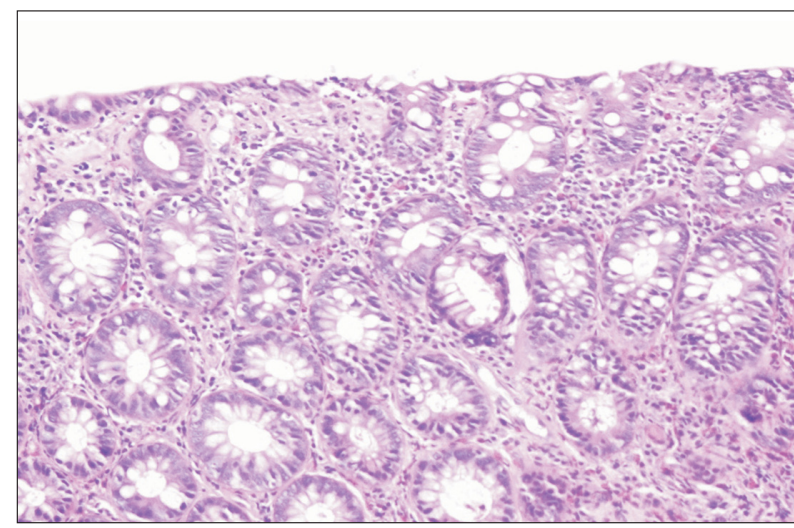

Fig. 1. Lymphocytic colitis. Images of $\mathrm{H} \& \mathrm{E}$ staining showing intraepithelial lymphocytosis and a preserved colonic architecture $(\times 20)$.

undergo a colonoscopy to evaluate the presence of chronic watery diarrhea. The endoscopic appearance unlike with IBD may be normal to near normal with rare cases of superficial mucosal cracking or nonspecific erythema. It is a combination of histologic changes with the proper clinical context that helps the pathologist to make the diagnosis histologically; however, histologic findings are not validated nor pathognomonic. There is controversy regarding where and how many biopsies to obtain at time of colonoscopy. According to the American Society for Gastrointestinal Endoscopy (ASGE), it is suggested that two or more biopsies are obtained from the right, transverse, descending, and sigmoid colon if colonoscopy is done or two or more biopsies are taken from the transverse colon, sigmoid colon, and descending colon if flexible sigmoidoscopy is done (ASGE 2013). ${ }^{49}$ However, societal guidelines are not evidenced based, rely on expert opinion, and have not been validated prospectively. The diagnostic yield of colonoscopy in patients with chronic diarrhea ranges from $7 \%$ to $32 \%{ }^{49}$ It is the combination of clinical symptoms with histologic changes that yields a diagnosis of MC.

\section{Diagnosis: histology}

Table 2 demonstrates the histologic definition of LC and CC. LC is defined by the presence of intraepithelial lymphocytes (>20/100 colonic surface epithelial cells), normal colonic architecture, lamina propria diffuse inflammation, and surface epithelial damage (Fig. 1). ${ }^{1,50-53}$ The lamina propria inflammatory

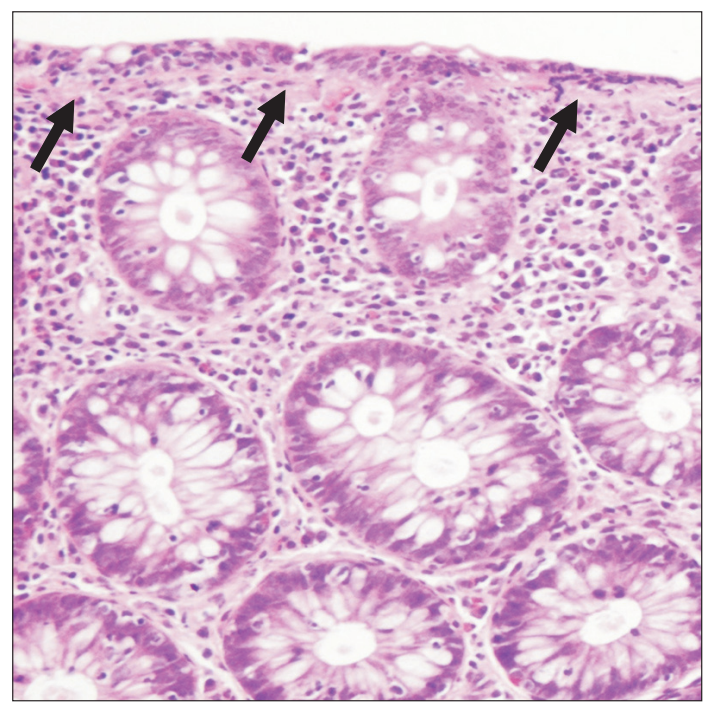

Fig. 2. Collagenous colitis. Images of H\&E staining showing a thickened subepithelial collagen band (arrows) and a preserved colonic architecture $(\times 40)$.

cellularity includes lymphocytes and plasma cells with rare but occasional neutrophils and eosinophils within the epithelium. ${ }^{1,50}$ Surface epithelial damage may include loss of mucin, flattening of columnar cells, and if severe enough "sloughing" of the surface epithelium. ${ }^{53,54}$ In severe cases the surface epithelium has also been termed "moth eaten." ${ }^{55}$

$\mathrm{CC}$ is defined by the presence of an abnormally thickened subepithelial collagen band (Fig. 2). ${ }^{54}$ For LC the subepithelial collagen band should be less than $10 \mu \mathrm{m}$, whereas this is greater than $10 \mu \mathrm{m}$ with CC (normal is 5 to $7 \mu \mathrm{m}$ ), which may be better seen with a trichrome stain. ${ }^{1,54,56}$ Within the collagen band, there may be capillaries, fibroblasts, eosinophils, inflammatory cells and increased numbers of lymphocytes. ${ }^{54}$ Similarly the lamina propria cellularity is increased. ${ }^{1}$ There may be an overlap with IBD features however when this occurs it is generally focal. In a sample of 150 patients with established MC (71 with LC and 79 with (C), pathologists found active crypt inflammation in 30\% with CC and 38\% with LC. ${ }^{52}$ Paneth cell metaplasia was present in 44\% with CC and 14\% with LC, which was associated with clinically more severe disease defined by abdominal pain and $>3$ stools per day. ${ }^{52}$

The collagen band has been studied and shown to not cor- 
relate with treatment or clinical response. In a randomized control trial with budesonide, there was no correlation between treatment and change in the mean thickness of the collagen band.$^{57}$ Prior investigations have also concluded that collagen table thickening does not correlate with duration of symptoms. ${ }^{58}$ Further, direct measurement of the band does not correlate with accuracy of diagnosis. ${ }^{35}$

Many MC randomized control trials have used limited histologic endpoints with a 0 to 3 scoring system of inflammation of the lamina propria, ${ }^{59,60}$ collagen band thickness $(>10 \mu \mathrm{m})$, presence of intraepithelial lymphocytosis, and damage to the surface epithelium. ${ }^{57}$

A 6-week randomized controlled trial of patients with CC were randomized to budesonide versus placebo and defined histologic improvement in two of the three categories: collagen band thickness, lamina propria inflammation (0 to 3), and presence or absence of surface epithelial degeneration. ${ }^{58}$ At the conclusion of 6 weeks of treatment, histologic improvement was noted in $60.9 \%$ of those treated with budesonide compared to $4.5 \%$ in the placebo group with no significant change in the collagen band. ${ }^{58}$ Furthermore, a randomized double-blind, placebo-controlled, multicenter study evaluating efficacy of budesonide as a maintenance treatment in CC also included histologic parameters. ${ }^{61}$ Patients had mucosal biopsies at baseline and 6 months after budesonide treatment for those who were in remission. ${ }^{61}$ Histologic findings included collagen band thickness, lamina propria inflammation (none, mild, moderate, severe) and presence or absence of surface epithelial damage. ${ }^{61}$ After 6 months of maintenance treatment, 93\% also demonstrated histologic improvement with no evidence of lamina propria inflammation at time of follow up in $80 \%$ of those treated with budesonide. ${ }^{61}$ Currently, further validated endpoints are needed to stratify natural history and assess response, beyond using standard histologic criteria as a lone response parameter.

\section{Quality of life}

In a case control study of patients with MC, impaired quality of life was demonstrated in both active CC and LC including impact on function in daily living, disease related worry, and wellbeing. ${ }^{21}$ In a population based study of 116 patients with CC from Hjortswang et al. ${ }^{62}$ quality of life is overall worse in those with active disease, including symptom burden, well-being, and social function when compared to those in clinical remission; to note, physical function was not impacted. Patients in clinical remission have overall health related quality of life scores similar to the comparison control population. ${ }^{62}$ Prior studies have also examined the impact of quality of life on CC. ${ }^{61,63,64}$ The Gastrointestinal Quality of Life Index (GIQLI) was also measured in a study that randomized patients with $\mathrm{CC}$ to budesonide versus placebo. ${ }^{63}$ After 6 weeks of treatment, patients overall GIQLI score improved including emotional and physical function. ${ }^{63}$ Baseline quality of life data approaches that of IBD. ${ }^{61}$ Thus, quality of life is an important consideration when treating MC with need to prevent any delays in diagnosis as studies have shown that successful treatment leads to improvement in patient's quality of life.

\section{Management}

There are multiple approaches to the treatment of MC depending on the physician's perspective of clinical disease severity. Given the questionable role of medications in the pathogenesis of MC, we first recommend potentially removing the inciting medication, specifically NSAIDs, PPIs, SSRIs and statins, if possible. ${ }^{19,65}$ Regardless of causation or association, multiple medications may cause diarrhea as a side effect of the medication. ${ }^{19}$

For clinically mild disease, antidiarrheal medications such as cholestyramine, loperamide, diphenoxylate/atropine may be used on an as needed or scheduled basis, particularly in patients who have an IBS overlap with MC. ${ }^{65}$ Studies suggest that bismuth subsalicylate may also be used as a treatment. ${ }^{66}$ In a small

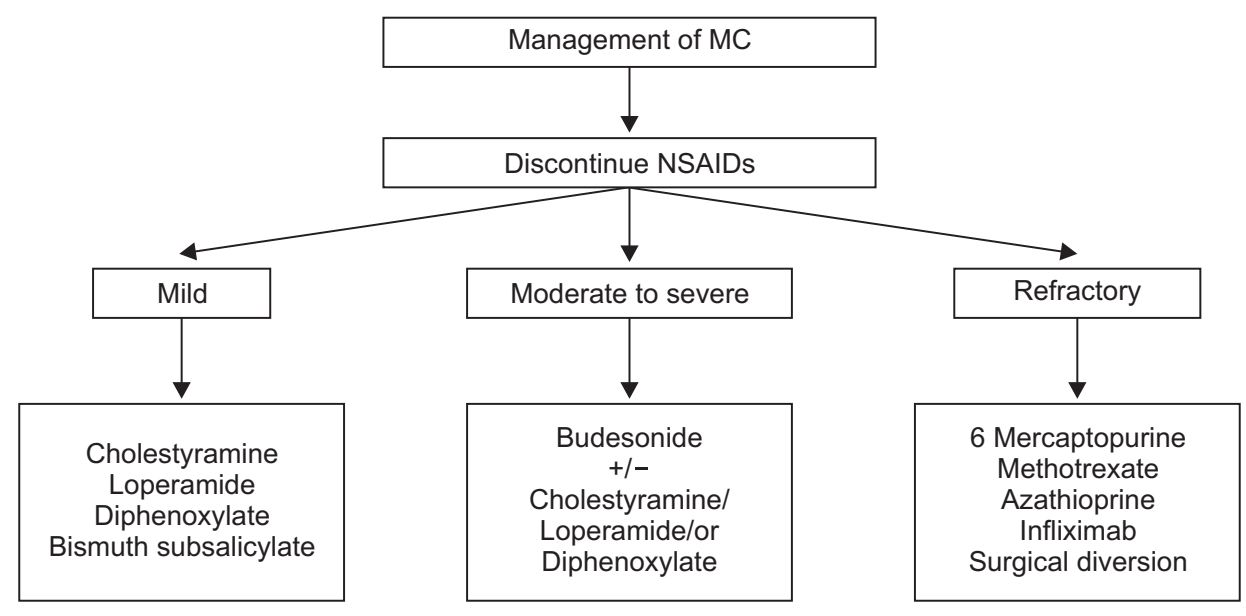

Fig. 3. Management of microscopic colitis (MC) according to symptom severity. NSAIDs, nonsteroidal anti-inflammatory drugs. 
study of 12 patients treated with eight tablets of bismuth subsalicylate daily for 8 weeks, 92\% had a symptomatic response within 2 weeks and 75\% had complete resolution of their symptoms. ${ }^{67}$ However, patients should be advised that stools may turn a darker color with bismuth. Aminosalicylates have also been proposed in the treatment of MC, however in a recent randomized trial with budesonide, mesalamine did no better than placebo for induction treatment in MC. ${ }^{41,65,68}$ Our proposed treatment algorithm is shown in Fig. 3.

The majority of treatment data is with corticosteroids due to their efficacy. In a cohort of 74 patients treated with corticosteroids in Olmsted County with a median follow-up of 4 years, better response rates were noted with budesonide compared to prednisone (82.5\% vs 52.9\%; OR, 4.18; 95\% CI, 1.3 to 13.5 ) with decreased rates of recurrence. ${ }^{69}$ Although multiple randomized trials have demonstrated response rates to corticosteroids, there are also high recurrence rates post treatment. ${ }^{57,58,60,61,70}$ In the above described cohort, up to 70\% of patients overall had a recurrence once corticosteroid therapy was completed. ${ }^{69}$ A recent meta-analysis supports this finding and evaluated both short and long term corticosteroid efficacy. ${ }^{71}$ Therefore data is needed on maintenance therapy as these patients offer recur post corticosteroid remission.

There is a small group of patients who will have steroid dependent disease for whom we recommend either maintenance budesonide versus consideration of step up therapy with immunomodulator therapy such as azathioprine, methotrexate, or 6 mercaptopurine..$^{23,72,73}$ Before deciding that a patient is medically refractory to initial treatments, we recommend screening for CD, excluding potential inciting medications such as NSAIDS, ruling out infection, as well as potentially repeating colonoscopy with biopsy to determine if MC still exists. ${ }^{23}$ Data on maintenance therapy is limited. In a maintenance trial of budesonide $6 \mathrm{mg}$ for 6 months versus placebo, decreased rates of relapse were seen with budesonide compared to placebo (26\% vs 65\%). ${ }^{61}$ In a 1 year study to address maintenance use of budesonide as a maintenance therapy, patients were randomized to an 8-week open label induction phase with histologic confirmation, and those in clinical remission then double blinded and randomized to receive a mean $4.5 \mathrm{mg}$ daily. ${ }^{64} 84.5 \%$ achieved clinical remission in the induction phase, with $61.4 \%$ maintaining remission in the budesonide group compared to $16.7 \%$ in the placebo group. ${ }^{64}$ Data on the use of methotrexate is limited. ${ }^{19}$ In a retrospective study of 43 patients, 19 were treated with oral methotrexate and 16 of 19 had partial to full response. ${ }^{74}$ However, other studies noted high rates of intolerance to methotrexate. ${ }^{75}$

For the further small subset of patients who are refractory to corticosteroid therapy, anti-tumor necrosis factor therapy may be an alternative. ${ }^{65}$ In a descriptive study of five patients with severe refractory MC, four of five were treated with infliximab with symptom improvement after one dose. ${ }^{76}$ In this population, one lost response to infliximab and transitioned to adalimumab, one had an allergic reaction to infliximab and was switched to adalimumab and one remained on infliximab with symptomatic improvement in daily bowel movements; however, the patient who had an allergic reaction to infliximab ultimately required a colectomy at 3 months due to loss of response. ${ }^{76}$ In the most severe or refractory cases, surgical diversion has shown to reverse colonic histology, so a diverting ostomy versus a colectomy versus ileal pouch anal anastomosis may be considered. ${ }^{59,76}$

\section{CONCLUSIONS}

MC is a chronic condition impacting quality of life resulting in chronic diarrhea, intermittent remission and relapses. However, once diagnosed, most cases of MC can be treated successfully with medication and improvement in quality of life. There are multiple challenges in the diagnosis and management of MC, including finding an optimal long term maintenance strategy for treatment, given that recurrence is common. There is also a need for better histologic criteria to define disease activity and response, and perhaps to help predict an individual patient's clinical course. These criteria would also serve as a meaningful guide for treatment algorithms and endpoints for clinical trials.

\section{CONFLICTS OF INTEREST}

No potential conflict of interest relevant to this article was reported.

\section{REFERENCES}

1. Münch A, Langner C. Microscopic colitis: clinical and pathologic perspectives. Clin Gastroenterol Hepatol 2015;13:228-236.

2. Fernández-Bañares F, Esteve M, Viver JM. Epidemiology of microscopic colitis. Gut 2007;56:1033.

3. Gentile NM, Khanna S, Loftus EV Jr, et al. The epidemiology of microscopic colitis in Olmsted County from 2002 to 2010: a population-based study. Clin Gastroenterol Hepatol 2014;12:838-842.

4. Pardi DS, Loftus EV Jr, Smyrk TC, et al. The epidemiology of microscopic colitis: a population based study in Olmsted County, Minnesota. Gut 2007;56:504-508.

5. Bonderup OK, Wigh T, Nielsen GL, Pedersen L, Fenger-Grøn M. The epidemiology of microscopic colitis: a 10-year pathologybased nationwide Danish cohort study. Scand J Gastroenterol 2015;50:393-398.

6. Fernández-Bañares F, Salas A, Esteve M, et al. Evolution of the incidence of collagenous colitis and lymphocytic colitis in Terrassa, Spain: a population-based study. Inflamm Bowel Dis 2011;17:10151020.

7. Tong J, Zheng Q, Zhang C, Lo R, Shen J, Ran Z. Incidence, prevalence, and temporal trends of microscopic colitis: a systematic review and meta-analysis. Am J Gastroenterol 2015;110:265-276.

8. Masclee GM, Coloma PM, Kuipers EJ, Sturkenboom MC. Increased 
risk of microscopic colitis with use of proton pump inhibitors and non-steroidal anti-inflammatory drugs. Am J Gastroenterol 2015;110:749-759.

9. Nielsen OH, Vainer B, Schaffalitzky de Muckadell OB. Microscopic colitis: a missed diagnosis? Lancet 2004;364:2055-2057.

10. Beaugerie L, Pardi DS. Review article: drug-induced microscopic colitis-proposal for a scoring system and review of the literature. Aliment Pharmacol Ther 2005;22:277-284.

11. Fernández-Bañares F, Esteve M, Espinós JC, et al. Drug consumption and the risk of microscopic colitis. Am J Gastroenterol 2007;102:324-330.

12. Bonderup OK, Fenger-Grøn M, Wigh T, Pedersen L, Nielsen GL. Drug exposure and risk of microscopic colitis: a nationwide Danish case-control study with 5751 cases. Inflamm Bowel Dis 2014;20:1702-1707.

13. Casella G, Villanacci V, Fisogni S, et al. Colonic left-side increase of eosinophils: a clue to drug-related colitis in adults. Aliment Pharmacol Ther 2009;29:535-541.

14. Gleeson MH, Davis AJ. Non-steroidal anti-inflammatory drugs, aspirin and newly diagnosed colitis: a case-control study. Aliment Pharmacol Ther 2003;17:817-825.

15. Levy AM, Yamazaki K, Van Keulen VP, et al. Increased eosinophil infiltration and degranulation in colonic tissue from patients with collagenous colitis. Am J Gastroenterol 2001;96:1522-1528.

16. Mahid SS, Minor KS, Soto RE, Hornung CA, Galandiuk S. Smoking and inflammatory bowel disease: a meta-analysis. Mayo Clin Proc 2006;81:1462-1471.

17. Yen EF, Pokhrel B, Du H, et al. Current and past cigarette smoking significantly increase risk for microscopic colitis. Inflamm Bowel Dis 2012;18:1835-1841.

18. Vigren L, Sjöberg K, Benoni C, et al. Is smoking a risk factor for collagenous colitis? Scand J Gastroenterol 2011;46:1334-1339.

19. Münch A, Aust D, Bohr J, et al. Microscopic colitis: current status, present and future challenges: statements of the European Microscopic Colitis group. J Crohns Colitis 2012;6:932-945.

20. Pardi DS, Kelly CP. Microscopic colitis. Gastroenterology 2011;140:1155-1165.

21. O'Toole A. Optimal management of collagenous colitis: a review. Clin Exp Gastroenterol 2016;9:31-39.

22. Bjørnbak C, Engel PJ, Nielsen PL, Munck LK. Microscopic colitis: clinical findings, topography and persistence of histopathological subgroups. Aliment Pharmacol Ther 2011;34:1225-1234.

23. Storr MA. Microscopic colitis: epidemiology, pathophysiology, diagnosis and current management-an update 2013. ISRN Gastroenterol 2013;2013:352718.

24. Kamp EJ, Kane JS, Ford AC. Irritable bowel syndrome and microscopic colitis: a systematic review and meta-analysis. Clin Gastroenterol Hepatol 2016;14:659-668.e1.

25. Abboud R, Pardi DS, Tremaine WJ, Kammer PP, Sandborn WJ, Loftus EV Jr. Symptomatic overlap between microscopic colitis and irritable bowel syndrome: a prospective study. Inflamm Bowel Dis 2013;19:550-553.
26. Macaigne G, Lahmek P, Locher C, et al. Microscopic colitis or functional bowel disease with diarrhea: a French prospective multicenter study. Am J Gastroenterol 2014;109:1461-1470.

27. Hjortswang H, Tysk C, Bohr J, et al. Defining clinical criteria for clinical remission and disease activity in collagenous colitis. Inflamm Bowel Dis 2009;15:1875-1881.

28. Cotter TG, Binder M, Loftus EV Jr, et al. Development of a microscopic colitis disease activity index: a prospective cohort study. Gut 2018;67:441-446.

29. Pisani LF, Tontini GE, Vecchi M, Pastorelli L. Microscopic colitis: what do we know about pathogenesis? Inflamm Bowel Dis 2016;22:450-458.

30. Bo-Linn GW, Vendrell DD, Lee E, Fordtran JS. An evaluation of the significance of microscopic colitis in patients with chronic diarrhea. J Clin Invest 1985;75:1559-1569.

31. Bürgel N, Bojarski C, Mankertz J, Zeitz M, Fromm M, Schulzke JD. Mechanisms of diarrhea in collagenous colitis. Gastroenterology 2002;123:433-443.

32. Stampfl DA, Friedman LS. Collagenous colitis: pathophysiologic considerations. Dig Dis Sci 1991;36:705-711.

33. Protic M, Jojic N, Bojic D, et al. Mechanism of diarrhea in microscopic colitis. World J Gastroenterol 2005;11:5535-5539.

34. Bohr J, Järnerot G, Tysk C, Jones I, Eriksson S. Effect of fasting on diarrhoea in collagenous colitis. Digestion 2002;65:30-34.

35. Lee E, Schiller LR, Vendrell D, Santa Ana CA, Fordtran JS. Subepithelial collagen table thickness in colon specimens from patients with microscopic colitis and collagenous colitis. Gastroenterology 1992;103:1790-1796

36. Ung KA, Gillberg R, Kilander A, Abrahamsson H. Role of bile acids and bile acid binding agents in patients with collagenous colitis. Gut 2000;46:170-175.

37. Zeroogian JM, Chopra S. Collagenous colitis and lymphocytic colitis. Annu Rev Med 1994;45:105-118.

38. Järnerot G, Bohr J, Tysk C, Eriksson S. Faecal stream diversion in patients with collagenous colitis. Gut 1996;38:154-155.

39. Veress B, Löfberg R, Bergman L. Microscopic colitis syndrome. Gut 1995;36:880-886.

40. Andresen L, Jørgensen VL, Perner A, Hansen A, Eugen-Olsen J, Rask-Madsen J. Activation of nuclear factor kappabeta in colonic mucosa from patients with collagenous and ulcerative colitis. Gut 2005;54:503-509.

41. Bohr J, Tysk C, Eriksson S, Abrahamsson H, Järnerot G. Collagenous colitis: a retrospective study of clinical presentation and treatment in 163 patients. Gut 1996;39:846-851.

42. Stewart M, Andrews CN, Urbanski S, Beck PL, Storr M. The association of coeliac disease and microscopic colitis: a large population-based study. Aliment Pharmacol Ther 2011;33:1340-1349.

43. Green PH, Yang J, Cheng J, Lee AR, Harper JW, Bhagat G. An association between microscopic colitis and celiac disease. Clin Gastroenterol Hepatol 2009;7:1210-1216.

44. Park T, Cave D, Marshall C. Microscopic colitis: a review of etiology, treatment and refractory disease. World J Gastroenterol 
2015;21:8804-8810.

45. Koskela RM, Karttunen TJ, Niemelä SE, Lehtola JK, Ilonen J, Karttunen RA. Human leucocyte antigen and TNFalpha polymorphism association in microscopic colitis. Eur J Gastroenterol Hepatol 2008;20:276-282.

46. Fernández-Bañares F, Esteve M, Farré C, et al. Predisposing HLADQ2 and HLA-DQ8 haplotypes of coeliac disease and associated enteropathy in microscopic colitis. Eur J Gastroenterol Hepatol 2005;17:1333-1338.

47. Fine KD, Do K, Schulte K, et al. High prevalence of celiac spruelike HLA-DQ genes and enteropathy in patients with the microscopic colitis syndrome. Am J Gastroenterol 2000;95:1974-1982.

48. Garner C, Ahn R, Ding YC, et al. Genome-wide association study of celiac disease in North America confirms FRMD4B as new celiac locus. PLoS One 2014;9:e101428.

49. ASGE Standards of Practice Committee, Sharaf RN, Shergill AK, et al. Endoscopic mucosal tissue sampling. Gastrointest Endosc 2013;78:216-224.

50. Langner C, Aust D, Ensari A, et al. Histology of microscopic colitis-review with a practical approach for pathologists. Histopathology 2015;66:613-626.

51. Fiehn AM, Bjørnbak C, Warnecke M, Engel PJ, Munck LK. Observer variability in the histopathologic diagnosis of microscopic colitis and subgroups. Hum Pathol 2013;44:2461-2466.

52. Ayata G, Ithamukkala S, Sapp H, et al. Prevalence and significance of inflammatory bowel disease-like morphologic features in collagenous and lymphocytic colitis. Am J Surg Pathol 2002;26:1414-1423.

53. Mahajan D, Goldblum JR, Xiao SY, Shen B, Liu X. Lymphocytic colitis and collagenous colitis: a review of clinicopathologic features and immunologic abnormalities. Adv Anat Pathol 2012;19:28-38.

54. Carmack SW, Lash RH, Gulizia JM, Genta RM. Lymphocytic disorders of the gastrointestinal tract: a review for the practicing pathologist. Adv Anat Pathol 2009;16:290-306.

55. Yen EF, Pardi DS. Review article: microscopic colitis--lymphocytic, collagenous and 'mast cell' colitis. Aliment Pharmacol Ther 2011;34:21-32.

56. Limsui D, Pardi DS, Smyrk TC, et al. Observer variability in the histologic diagnosis of microscopic colitis. Inflamm Bowel Dis 2009;15:35-38.

57. Bonderup OK, Hansen JB, Birket-Smith L, Vestergaard V, Teglbjaerg PS, Fallingborg J. Budesonide treatment of collagenous colitis: a randomised, double blind, placebo controlled trial with morphometric analysis. Gut 2003;52:248-251.

58. Miehlke S, Heymer P, Bethke B, et al. Budesonide treatment for collagenous colitis: a randomized, double-blind, placebo-controlled, multicenter trial. Gastroenterology 2002;123:978-984.

59. Yen EF, Pardi DS. Review of the microscopic colitides. Curr Gastroenterol Rep 2011;13:458-464.

60. Bonderup OK, Hansen JB, Teglbjaerg PS, Christensen LA, Fallingborg JF. Long-term budesonide treatment of collagenous colitis: a randomised, double-blind, placebo-controlled trial. Gut 2009;58:68-72.

61. Miehlke S, Madisch A, Bethke B, et al. Oral budesonide for maintenance treatment of collagenous colitis: a randomized, doubleblind, placebo-controlled trial. Gastroenterology 2008;135:15101516.

62. Hjortswang H, Tysk C, Bohr J, et al. Health-related quality of life is impaired in active collagenous colitis. Dig Liver Dis 2011;43:102109.

63. Madisch A, Heymer P, Voss C, et al. Oral budesonide therapy improves quality of life in patients with collagenous colitis. Int $\mathrm{J}$ Colorectal Dis 2005;20:312-316.

64. Münch A, Bohr J, Miehlke S, et al. Low-dose budesonide for maintenance of clinical remission in collagenous colitis: a randomised, placebo-controlled, 12-month trial. Gut 2016;65:47-56.

65. Yen EF, Pardi DS. Non-IBD colitides (eosinophilic, microscopic). Best Pract Res Clin Gastroenterol 2012;26:611-622.

66. Amaro R, Poniecka A, Rogers AI. Collagenous colitis treated successfully with bismuth subsalicylate. Dig Dis Sci 2000;45:14471450.

67. Fine KD, Lee EL. Efficacy of open-label bismuth subsalicylate for the treatment of microscopic colitis. Gastroenterology 1998;114:2936.

68. Olesen M, Eriksson S, Bohr J, Järnerot G, Tysk C. Lymphocytic colitis: a retrospective clinical study of 199 Swedish patients. Gut 2004;53:536-541.

69. Gentile NM, Abdalla AA, Khanna S, et al. Outcomes of patients with microscopic colitis treated with corticosteroids: a populationbased study. Am J Gastroenterol 2013;108:256-259.

70. Miehlke S, Madisch A, Voss C, et al. Long-term follow-up of collagenous colitis after induction of clinical remission with budesonide. Aliment Pharmacol Ther 2005;22:1115-1119.

71. Stewart MJ, Seow CH, Storr MA. Prednisolone and budesonide for short- and long-term treatment of microscopic colitis: systematic review and meta-analysis. Clin Gastroenterol Hepatol 2011;9:881890.

72. Pardi DS, Ramnath VR, Loftus EV Jr, Tremaine WJ, Sandborn WJ. Lymphocytic colitis: clinical features, treatment, and outcomes. Am J Gastroenterol 2002;97:2829-2833.

73. Pardi DS, Loftus EV Jr, Tremaine WJ, Sandborn WJ. Treatment of refractory microscopic colitis with azathioprine and 6-mercaptopurine. Gastroenterology 2001;120:1483-1484.

74. Riddell J, Hillman L, Chiragakis L, Clarke A. Collagenous colitis: oral low-dose methotrexate for patients with difficult symptoms: long-term outcomes. J Gastroenterol Hepatol 2007;22:1589-1593.

75. Münch A, Bohr J, Vigren L, Tysk C, Ström M. Lack of effect of methotrexate in budesonide-refractory collagenous colitis. Clin Exp Gastroenterol 2013;6:149-152.

76. Esteve M, Mahadevan U, Sainz E, Rodriguez E, Salas A, Fernández-Bañares F. Efficacy of anti-TNF therapies in refractory severe microscopic colitis. J Crohns Colitis 2011;5:612-618. 